\title{
BMJ Open Effectiveness and cost-effectiveness of duloxetine added to usual care for patients with chronic pain due to hip or knee osteoarthritis: protocol of a pragmatic open-label cluster randomised trial (the DUO trial)
}

Jacoline J van den Driest, ${ }^{1}$ Dieuwke Schiphof, ${ }^{1}$ Pim A J Luijsterburg, ${ }^{1}$ Aafke R Koffeman, ${ }^{2}$ Marc A Koopmanschap, ${ }^{3}$ Patrick J E Bindels, ${ }^{1}$ Sita M A Bierma-Zeinstra ${ }^{1,6}$

To cite: van den Driest JJ, Schiphof D, Luijsterburg PAJ, et al. Effectiveness and costeffectiveness of duloxetine added to usual care for patients with chronic pain due to hip or knee osteoarthritis: protocol of a pragmatic openlabel cluster randomised trial (the DUO trial). BMJ Open 2017;7:e018661. doi:10.1136/ bmjopen-2017-018661

- Prepublication history for this paper is available online. To view these files please visit the journal online (http://dx.doi. org/10.1136/bmjopen-2017018661)

Received 13 July 2017 Revised 4 August 2017 Accepted 10 August 2017

CrossMark

For numbered affiliations see end of article.

Correspondence to Jacoline J van den Driest; j.vandendriest@erasmusmc.nl

\section{ABSTRACT}

Introduction 0steoarthritis $(\mathrm{OA})$ is a highly prevalent painful condition of the musculoskeletal system. The effectiveness of current analgesic options has proven to be limited and improved analgesic treatment is needed. Several randomised placebo-controlled trials have now demonstrated the efficacy of duloxetine, an antidepressant with a centrally acting effect, in the treatment of $\mathrm{OA}$ pain. The aim of the current study is to investigate if duloxetine is effective and cost-effective as a third-choice analgesic added to usual care for treating chronic pain compared with usual care alone in general practice.

Methods and analysis A pragmatic open, cluster randomised trial is conducted. Patients with pain due to hip or knee OA on most days of the past 3 months with insufficient benefit of non-steroidal anti-inflammatory drugs or contraindications or intolerable side effects are included. General practices are randomised to either (1) duloxetine and usual care or (2) usual care only. Primary outcome is pain at 3 months measured on the Western Ontario and McMaster Universities Osteoarthritis Index (WOMAC) pain subscale. Secondary outcomes at 3 months and 1 year are pain (WOMAC, at 1 year), function (WOMAC), adverse reactions, quality of life and modification of the response to treatment by the presence of centrally sensitised pain (modified PainDETECT). At 1 year, medical and productivity costs will be assessed. Analyses will be performed following the intention-to-treat principle taking the cluster design into account.

Ethics and dissemination The study is approved by the local Medical Ethics Committee (2015-293). Results will be published in a scientific peer-reviewed journal and will be communicated at conferences.

Trial registration number Dutch Trial Registry(ntr4798); Pre-results.

\section{BACKGROUND AND RATIONALE}

Osteoarthritis (OA) is a highly prevalent chronic condition of the musculoskeletal

\section{Strengths and limitations of this study}

- This is the first study that investigates the effectiveness and cost-effectiveness of duloxetine as a third-choice analgesic in primary care.

- The efficacy of duloxetine has been demonstrated, but the effectiveness and cost-effectiveness are not yet known. The pragmatic open-label stratified cluster design of this trial is particularly appropriate to study the effectiveness of this treatment strategy in real-life clinical practice.

- This is the first study that assesses if the effectiveness of duloxetine is predominantly found in those patients suffering from centrally sensitised pain.

- The cluster randomised design can be prone to recruitment bias, though we aim to minimise this bias and maximise generalisability by identifying all eligible patients before randomisation.

- Stratified randomisation for important GP practice characteristics is used to minimise the possible baseline imbalance between randomised groups.

system. Approximately $15 \%$ of the population suffers from OA. Among elderly, the lifetime risk for knee OA is $40 \%$ in men and $47 \%$ in women ${ }^{12}$ and it is the single-most contributing factor to a decreased physical well-being. ${ }^{3}$ It is predicted that OA will become the fourth leading cause of disability worldwide by the year $2020 .{ }^{4}$ The majority of patients suffering from $\mathrm{OA}$ are treated in primary care. ${ }^{56}$ The general practitioner (GP) plays a key role in the treatment of symptomatic pain, the most debilitating symptom of this condition. Currently, usual care by the GP consists of education, referral 
for exercise therapy and prescribing symptomatic pain medication using a stepwise approach. The first step consists of paracetamol. If its analgesic effect proves insufficient, non-steroidal anti-inflammatory drugs (NSAIDs) can be prescribed as a second step, and subsequently tramadol or other opioids. ${ }^{7}$ So far, however, the effectiveness of symptomatic pain treatment has proven to be limited ${ }^{8-10}$ and the majority of the patients still report pain despite taking pain medication. ${ }^{11}{ }^{12}$ In addition, these analgesics are often contraindicated, especially in elderly patients, and they are associated with the occurrence of serious adverse reactions. Improved analgesic treatment or a new approach to OA pain treatment is therefore needed, especially since there are no treatment options available aimed at delaying or halting the process of OA. Surgical joint replacement is the exception but is an intervention which is costly, does not always lead to a satisfied results for patients and is also of limited durability. ${ }^{413}$ An effective and relatively safe medicine, when current options fail, could improve the quality of life for these patients with chronic pain. In the long term, it could potentially help postpone the need for a joint replacement and revision surgery while retaining quality of life.

Traditionally, pain in OA was assumed to consist solely of nociceptive pain due to inflammation, degradation and remodelling of joint tissue. However, it has since been demonstrated that in addition to nociceptive pain, peripheral sensitised pain from locally generated inflammatory factors and centrally sensitised pain can be present in OA. ${ }^{14}$ Intense, repeated or prolonged nociceptive input can lead to hyperexcitability of pain circuits in the central nervous system. ${ }^{15}$ Normally, descending inhibitory pathways from the brainstem modulate pain processing through the release of norepinephrine and serotonin. These inhibitory pathways can be impaired in patients with OA. ${ }^{15}$ This disinhibition of descending pathways further contributes to central sensitisation in OA. Centrally sensitised pain is present in a substantial percentage $(30 \%-37 \%)^{16} 17$ of patients with chronic pain due to OA and is thought to respond particularly poorly to currently prescribed analgesics, as it requires medication with a centrally acting agent. Duloxetine is a serotonin-norepinephrine reuptake inhibitor and acts centrally by strengthening the inhibition of the descending pathways.

Several randomised placebo-controlled trials have now demonstrated the efficacy of low-dose duloxetine versus placebo in the treatment of pain in OA (clinically relevant effect sizes of $0.4-0.5$ for pain in $\mathrm{OA}^{1819}$ and 0.6 for disability in $\mathrm{OA}^{18}$ ) and the most recent guideline of the Osteoarthritis Research Society International (OARSI) now recommend duloxetine for the non-surgical management of knee OA. ${ }^{10}$ These trials are shortterm randomised placebo-controlled trials in a highly controlled and secondary care setting. The effectiveness of duloxetine as third-choice analgesia in general practice is not known. Neither is clear whether the effectiveness of duloxetine is predominantly found in those patients suffering from pain with characteristics of central sensitisation, or whether duloxetine as thirdchoice analgesia in general practice is cost-effective, if found effective.

\section{METHODS AND DESIGN Objectives}

The primary objective of this study is to investigate whether duloxetine added to usual care is effective as third-choice pain medication (when NSAIDs fail, are contraindicated or have intolerable side-effects) in reducing chronic pain Western Ontario and McMaster Universities Osteoarthritis Index (WOMAC) in primary care patients with OA after 3 months, compared with usual care alone.

Secondary objectives are (1) to assess whether the presence of characteristics of central sensitisation (modified painDETECT $>12.0$ ) favourably modifies the response to treatment with duloxetine (WOMAC pain at 3 months and WOMAC pain and function at 1 year) and (2) to examine the cost-effectiveness of treatment with duloxetine at 1 year.

\section{Study design}

A pragmatic open-label cluster randomised controlled trial with a follow-up of 1 year is conducted (figure 1). To avoid contamination between the two intervention groups, randomisation takes place on the level of the GP practice. Patients, researchers and GPs are not blinded to the assigned treatment.

\section{Randomisation}

GP practices are randomised to the duloxetine or usual care group. Since provided care can differ based on practice characteristics, stratification is performed on: (1) socioeconomic status of the practice location (low socioeconomic status vs normal and high socioeconomic status, based on the registration by the Netherlands Institute for Social Research $\left.{ }^{20}\right)$; (2) the number of GPs working at the practice (one full-time equivalent or smaller vs larger than one full-time equivalent) and (3) the mean age of the GPs per practice (younger than 50 years or 50 years and older). ${ }^{21-23}$ The randomisation sequence is determined by an independent data manager from the department, with the use of a computer-generated randomisation list. A block design with blocks random varying between two and six is used to equally balance the groups. The randomisation procedure is concealed to the involved researchers of the study. Randomisation of the GP practice is performed after eligible patients are identified and the first patient of the GP practice has signed informed consent.

\section{Inclusion criteria}

All patients must be aged 18 years or older and have hip and/or knee OA based on the clinical American College of Rheumatology criteria. ${ }^{24}$ Chronic pain 


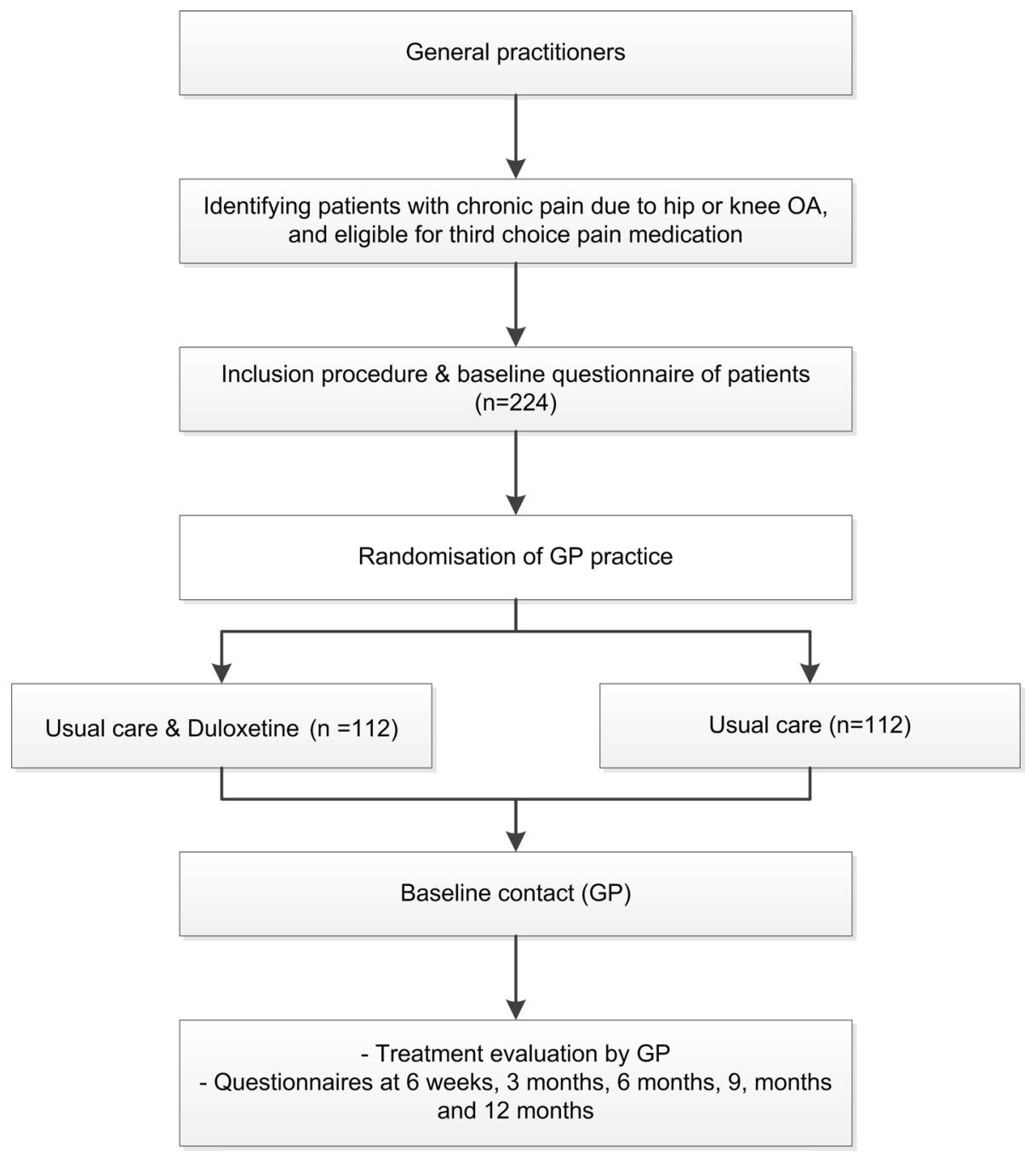

Figure 1 Flow chart of the study. GP, general practitioner; OA, osteoarthritis.

should be present, which is defined as pain on most days during the last 3 months. In addition, patients must have insufficient benefit of NSAIDs, contraindications for NSAIDs or previous adverse reactions to NSAIDs (ie, eligible for third-choice pain medication).

\section{Exclusion criteria}

Patients who meet one of the following criteria will be excluded from the trial.

- Scheduled for a hip or knee replacement;

- Current use of antidepressants;

- Current use of neuropathic pain medication;

- Rheumatoid arthritis;

- Unable to sign informed consent;

- Contraindications for duloxetine (current use of monoamine oxidase inhibitors, uncontrolled narrowangle glaucoma, the combination with other central nervous acting drugs (eg, benzodiazepines), hypersensitivity to duloxetine, liver disease resulting in hepatic impairment, severe renal impairment (creatinine clearance $<30 \mathrm{~mL} / \mathrm{min}$ ), current use of strong CYP1A2 inhibitors, current use of CYP2D6 inhibitors and substrates, uncontrolled hypertension, pregnancy or lactation).

Initially, patients who currently used opioids or used these in the past 3 months were excluded from the trial, because opioids are already third-choice medication in the Netherlands. Though, when patients still feel pain despite the use of opioids, it may be that these complaints reflect a centrally sensitised pain, since this type of pain is thought to respond less well to traditional analgesics. Especially these patients can benefit from duloxetine. Therefore, these patients are also eligible.

\section{Intervention \\ Duloxetine}

Patients in the duloxetine arm are prescribed duloxetine $60 \mathrm{mg}$ once a day. During the first week of treatment, the patient starts with $30 \mathrm{mg}$ duloxetine per day to minimise potential adverse events due to duloxetine. If tolerated well, the dose is increased to $60 \mathrm{mg} /$ day in the second week. Therapeutic benefit of duloxetine is assessed regularly by the treating GP (after 2 weeks, 1, 3, 6, 9, 12 months and when necessary). If patients experience no effect of 
duloxetine after 3 months or patients experience intolerable side effects, duloxetine can gradually be discontinued. Patients also receive usual care according to the GP guidelines in the Netherlands, ${ }^{7}$ as described below.

\section{Usual care}

Patients are treated according to the current Dutch GP guideline which comprise education, life style advice, dietary therapy, physiotherapy and analgesics. ${ }^{7}$ Analgesics are prescribed according to the opinion and experience of the treating GP. Analgesic choice depends on what the GP and patients decide. Paracetamol and NSAIDs have failed in these patients. A GP can decide to prescribe or continue an opioid, or the treatment can remain the same. Depending on the comorbidities of the patients, contraindications and opinion of the GP other interventions are also allowed.

\section{Cointerventions}

Patients assigned to the usual care group cannot be prescribed duloxetine. In both groups, intra-articular injection with glucocorticoids or a referral for joint replacement is allowed, as these are in accordance with Dutch GP guidelines and appropriate within the pragmatic design of this trial.

\section{Study procedures}

Patients are recruited from general practices. GPs in the Rotterdam region are invited to participate. If a GP practice is held by more than one GP, all GPs are randomised into the same arm. In this way, contamination between the groups is prevented when a patient visits another GP in the same practice. Demographic characteristics of the GP practice are registered. Randomisation of the GP practice is performed after the first patient of the GP practice has signed the informed consent form.

All eligible patients are identified by an electronic search in the participating GPs medical records. The GP decides which patients receive an invitation for the trial. In this way, possible recruitment bias can be monitored. Subsequently, eligible patients are sent information about the current trial. If a patient is interested in participating, the patient is contacted by a member of the research team over telephone. During this contact, the study is explained and eligibility of the patient is re-evaluated. If a patient is interested and eligible, the patient receives further written information about the study. After 2 weeks, the patients is contacted a second time over telephone to obtain informed consent. After filling out the baseline questionnaire, patients are told to which intervention their GP practice is randomised. In both study arms, patients consult their GP for their intervention (figure 1). In the duloxetine arm, the GP prescribes duloxetine during the consultation. If at this point the patient decides not to take the duloxetine after all, the patient remains in the study in accordance with the pragmatic approach.

Patients receive follow-up questionnaires at 6 weeks, 3 , 6, 9 and 12 months (table 1) after filling out the baseline questionnaire. The questionnaire at 6 weeks is added to the initial protocol to monitor the therapeutic effect of duloxetine more closely in the first weeks.

\begin{tabular}{|c|c|c|c|c|c|c|}
\hline & Baseline & 6 weeks & 3months & 6 months & 9 months & 12 months \\
\hline \multicolumn{7}{|l|}{ Outcome measures } \\
\hline Pain score (WOMAC) & $\mathrm{x}$ & $\mathrm{x}$ & $\mathrm{x}$ & $\mathrm{x}$ & $\mathrm{x}$ & $\mathrm{x}$ \\
\hline Function score (WOMAC) & $x$ & $x$ & $x$ & $x$ & $x$ & $x$ \\
\hline HOOS/KOOS & $\mathrm{x}$ & $x$ & $\mathrm{x}$ & $\mathrm{x}$ & $\mathrm{x}$ & $\mathrm{x}$ \\
\hline Quality of life (EuroQol-5D-5L) & $\mathrm{x}$ & & $x$ & $\mathrm{x}$ & $x$ & $\mathrm{x}$ \\
\hline Medical costs (iMCQ) & $x$ & & $x$ & $x$ & $x$ & $x$ \\
\hline Productivity cost (iPCQ) & $x$ & & $x$ & $x$ & $x$ & $x$ \\
\hline Cointerventions & & $x$ & $x$ & $\mathrm{x}$ & $x$ & $\mathrm{x}$ \\
\hline Adverse events & & $x$ & $x$ & $\mathrm{x}$ & $x$ & $\mathrm{x}$ \\
\hline Compliance & $x$ & & $x$ & $\mathrm{x}$ & $x$ & $\mathrm{x}$ \\
\hline Patients satisfaction & & $x$ & $x$ & $x$ & $x$ & $x$ \\
\hline
\end{tabular}

\section{Others}

\begin{tabular}{llll} 
Demographic data & $\mathrm{X}$ & & \\
Comorbidities & $\mathrm{X}$ & & \\
Presence of centrally sensitised & $\mathrm{X}$ & $\mathrm{X}$ & $\mathrm{X}$ \\
pain (modified painDETECT) & $\mathrm{X}$ & $\mathrm{X}$ \\
\hline Presence of depression or anxiety (HADS) & & \\
\hline
\end{tabular}

HADS, Hospital and Anxiety Depression Scale; HOOS, Hip disability and Osteoarthritis Outcome Score; iMCQ, iMTA Medical Cost Questionnaire; iPCQ, iMTA Productivity Cost Questionnaire; KOOS, Knee Injury and Osteoarthritis Outcome Score; WOMAC, Western Ontario and McMaster University Osteoarthritis Index. 


\section{Safety}

All adverse events reported by the subject are recorded. All serious adverse events (SAEs) are reported to the medical ethics committee (MEC). All SAEs are monitored until resolution or stabilisation. No Data and Safety Monitoring Board is needed, since adverse events of duloxetine are well known and duloxetine is registered in the Netherlands for the treatment of depressive disorders and diabetic neuropathy. Monitoring of the study is carried out once a year because of the negligible risk profile (Standards of The Netherlands Federation of University Medical Centres).$^{25}$

\section{Outcome measures}

Primary outcome

The primary outcome is pain at 3-month follow-up measured with the WOMAC pain subscale. During the study period, patients complete the Knee Injury and Osteoarthritis Outcome Score (KOOS) or the Hip Disability and Osteoarthritis Outcome Score (HOOS). ${ }^{26}{ }^{27}$ The HOOS and KOOS consist of five subscales: pain, other symptoms, ADL function, function in sport and recreation, and knee/hip-related quality of life. The KOOS and HOOS are developed as an extension of the WOMAC score, with the purpose of evaluating both short-term and long-term symptoms and function. All WOMAC pain and function questions are included in their full and original forms in the KOOS/HOOS questionnaire. ${ }^{26}{ }^{28}$ Therefore, WOMAC scores can be calculated from the KOOS and HOOS scores. The questions apply to the previous week and each indicator is scored on a 5-point Likert scale $(0=$ extreme problems and $4=$ no problems). Scores range between 0 and 100, and higher scores indicate less symptoms.

\section{Secondary outcomes}

Secondary outcomes are pain at 1 year (WOMAC pain subscale) and disability at 1year (WOMAC function subscale). Adverse reactions and cointerventions for OA pain are systematically recorded in the follow-up questionnaires. Patients' satisfaction with the treatment is measured on an 11-point numerical rating scale $(0=$ completely dissatisfied, $10=$ completely satisfied $)$.

Health-related quality of life is measured with the EuroQol-5D (EQ-5D-5L) which comprises a descriptive system with five dimensions and a Visual Analogue Scale. ${ }^{29}$ Costs are measured using the iMTA Medical Consumption Questionnaire (iMCQ) and the iMTA Productivity Cost Questionnaire (iPCQ). The iMCQ is a non-disease-specific questionnaire to determine the direct medical and patients' costs of the previous 3 months. ${ }^{30}$ The iPCQ is focused on the indirect costs of the healthcare system; it measures the productivity losses in the last 4 weeks. ${ }^{31}$

Compliance to the treatment is estimated by questions about medication use (dose, how often each day, number of days). Furthermore, the Beliefs about Medication Questionnaire (BMQ) is asked. The BMQ consists of two sections: the BMQ-General and BMQ-Specific section ${ }^{32}$ assessing patients' thoughts about medicine in general and about OA medication specifically. Each section consists of two subscales. The BMQ-General contains the subscales Harm and Overuse (scores 4-20). The BMQ-Specific encompasses the subscales Necessity and Concerns (scores 5-25). Higher scores on the BMQ-Specific section are correlated with self-reported therapy adherence. ${ }^{33} 34$

The effect of the intervention will also be evaluated by the OMERACT-OARSI response criteria. These criteria are a uniform core set of outcome measurements for OA. Response is defined as (1) a high improvement in pain (WOMAC pain) or function (WOMAC function) $(\geq 50 \%)$ and an absolute change of $\geq 20$ (on an interval scale $0-100)$ or (2) improvement in at least two of the three following: pain $\geq 20 \%$ (WOMAC pain) and absolute change $\geq 10$; function $\geq 20 \%$ (WOMAC function) and absolute change $\geq 10$; patients' global assessment $\geq 20 \%$ and absolute change $\geq 10 .{ }^{35}$

\section{Other study outcomes}

At baseline the following patient characteristics are collected: age, gender, height and weight, education, comorbidities and the duration of complaints. In addition, patients fill out the modified painDETECT questionnaire which is adapted for patients with $\mathrm{OA}^{36}$ This questionnaire is validated for identifying "neuropathic-like' symptoms which correlate with signs of central sensitisation in OA. Higher modified painDETECT scores indicate the presence of central sensitisation (scores $>12$, range -1 to 35$).{ }^{16} 37$ The modified painDETECT was recently validated in Dutch for both patients with knee OA and for patients with hip OA. ${ }^{38}$

Finally, the Hospital Anxiety and Depression Scale is assessed to establish the analgesic effect of duloxetine independent of the effects on mood or anxiety. ${ }^{39}$

\section{Sample size}

To detect a clinically relevant difference in WOMAC pain of 1.9 points (pooled SD 4.8) ${ }^{18}$ between the two treatment groups with an effect size of 0.4 (power $80 \%$; alpha 0.05 ), taking into account the cluster randomisation with an assumption of three patients per GP and an intracluster correlation coefficient (ICC) of 0.01, 102 patients per treatment group are required. As we expect around $10 \%$ loss to follow-up, ${ }^{40}$ we need to include 224 in total (2×112). In order to detect an effect (with an effect size of 0.6 ) in the patients with centrally sensitised pain we need 44 patients per group (with the same power and cluster assumptions). In the patients who are currently included in the trial, centrally sensitised pain (PainDETECT score $>12$ ) is present in $47 \%$ of the included patients. This percentage is higher than in the general OA population $(37 \%) .{ }^{16}$ This over-representation is probably related to our inclusion and exclusion criteria. When we assume a presence of centrally sensitised pain in $47 \%$ of our included patients, 206 patients need to be included to answer the secondary objective. Therefore, no sample size adjustments have to be made. 
The current sample size is adjusted due to recruitment problems. In the initial sample size calculation, 362 patients needed to be included to detect a clinically relevant difference in WOMAC pain of 1.9 points. In this calculation, an ICC of 0.1 and five patients per GP were assumed. The inclusion of five patients per GP was not feasible and therefore this number was altered to three. Furthermore, the ICC of 0.1 was a conservative estimate and an ICC tends to be around 0.01 in general practice. ${ }^{41}$

\section{Data management}

All personal data are handled confidentially and anonymously. Each patient is allocated a unique code, which is used on the questionnaires and in the database. The link between the code and the patient identification is only be accessible to the researcher and the data manager. The software programs LimeSurvey and GemsTracker are used for the online questionnaires and patients' personal data, respectively.

\section{Patient involvement}

Within the department of General Practice a patient panel exists (Primeur Patient Panel), with patient representatives who are involved in all phases of research. ${ }^{42}$ Patient representatives from the Primeur Patient Panel, familiar with OA, have helped to design this study and will be involved in all phases of the study. They read and gave comments on the proposal, all patient information and procedures.

\section{Statistical analysis}

Descriptive statistics will be used to describe patient characteristics, baseline values of the outcome measures and compliance to the intervention. Primary (WOMAC pain at 3 months) and secondary (WOMAC pain and function at 1 year) outcomes will be analysed using generalised linear mixed models with repeated measurements. The stratified cluster randomisation will be taken into account when analysing the data (multilevel analysis). When baseline characteristics are statistical significantly different between the two treatment groups, we will perform a confounder analysis. If the effect on the outcome changes $10 \%$ or more the baseline characteristic will be considered a confounder and analyses will be adjusted accordingly. All analyses will be performed following the intentionto-treat principle. Additional per protocol analyses will also be carried out; for the primary outcome, WOMAC pain at 3 months, in the intervention arm patients who discontinued duloxetine (within 4 weeks after start) or did not start at all will not be considered, and patients in the control arm who were prescribed antidepressants will not be considered. Subgroup analyses to assess the effectiveness of treatment in patients with centrally sensitised pain will be completed in patients who score higher than 12 on the modified painDETECT. The same analysis as described earlier will be carried out.

To evaluate cost-effectiveness of usual care and duloxetine versus usual care only in patients with chronic pain due to hip or knee OA, a cost-effectiveness analysis will be performed using the primary outcome: pain measured with the WOMAC questionnaire. A cost-utility analysis will be performed to compare our study with other studies in OA research using a general accepted outcome: quality-adjusted life years (QALYs). To calculate QALYs based on the EQ-5D-5L, utility values of the Dutch public for EuroQol health states will be applied. ${ }^{43}$ Using non-parametric bootstrapping (randomly drawing 2500 observations with replacement from the patient sample), the degree of uncertainty for costs and health effects and the cost-utility ratio will be depicted in a cost-effectiveness plane.

In addition, an acceptability curve is drawn, which indicates the probability that the intervention has lower incremental cost per QALY gained than various thresholds for the maximum willingness to pay for an extra QALY.

Social perspective and healthcare perspective will be the basis of the economic analysis in which the direct and productivity costs in the groups will be compared using the iMCQ $^{30}$ and the Productivity Cost Questionnaire. ${ }^{31}$

\section{Spirit guidelines}

This protocol meets the SPIRIT guidelines for reporting on study protocols for clinical trials.

\section{DISSEMINATION}

Results will be communicated at international and national conferences and will be published in a scientific peer-reviewed journal. Together with the patient representatives of the Primeur Patient Panel, the results and ideas for dissemination will be discussed. They will check the validity of the conclusions from a public perspective and highlight findings that are more relevant to the public.

Substantial protocol amendments will be communicated to the MEC, the competent authority (Centrale Commissie Mensgebonden Onderzoek), ZonMw and cooperating GPs.

\section{DISCUSSION}

This study assesses the effectiveness and cost-effectiveness of duloxetine added to usual care for patients with chronic pain due to hip and/or knee OA in a primary care setting. As far as we know this is the first pragmatic trial in which duloxetine is compared directly with usual care in primary care to establish the effectiveness of treatment with duloxetine. Until now only the efficacy has been shown. ${ }^{18} 194445$ Longterm effects, the comparison with usual care and cost-effectiveness are unknown.

This pragmatic trial evaluates the use of duloxetine in everyday practice and has a high external validity due to the relatively unselected patients and flexible conditions. Therefore, the DUO trial will be helpful in answering the question whether the interventions has additional value in 'real life', ${ }^{46} 47$ 
We choose the cluster randomised design, because the design has particular utility in effectiveness and implementation studies. This design is particularly appropriate for the evaluation of interventions that naturally are applied at cluster level, for example, general practices that choose a certain stepped approach. ${ }^{47}$

Issues that require specific attention in this trial due to the cluster design are the generalisability, recruitment bias and possible baseline imbalances between the randomised groups. We monitored the risk of recruitment bias and maximised the generalisability by inviting all eligible patients from a cluster to participate in this study. Possible baseline imbalances are minimised by the stratification of the clusters.

Finally, it is not known whether the presence of centrally sensitised pain favourably modifies the treatment response to duloxetine. The modified painDETECT is a questionnaire that can be easily used in a primary care setting, in contrast to traditional extensive measurements such as quantitative sensory testing. ${ }^{16}$ Patients with knee OA with higher modified painDETECT scores $(>12)$ have higher odds of having signs of central sensitisation. ${ }^{16}$ If the presence of centrally sensitised pain does modify treatment response to duloxetine, this finding will enable more targeted treatment choices in primary care patients with OA.

In conclusion, the DUO trial is a cluster randomised trial to assess the effectiveness of duloxetine in patients with hip or knee OA with chronic pain in primary care. The trial will answer the question whether duloxetine can be used as third-choice pain medication in primary care and may enable more targeted treatment choices.

\section{Author affiliations \\ ${ }^{1}$ Department of General Practice, Erasmus University Medical Center, Rotterdam, The Netherlands \\ ${ }^{2}$ Department of Public Health and Primary Care, Leids Universitair Medisch Centrum, Leiden, Zuid-Holland, The Netherlands \\ ${ }^{3}$ Department of Health Policy and Management/iMTA, Erasmus University Rotterdam, Rotterdam, The Netherlands \\ ${ }^{6}$ Department of Orthopedics, Erasmus University Medical Center, Rotterdam, The Netherlands}

Contributors JJvdD, DS, PAJL, ARK, MAK, PJEB, SMAB-Z participated in the design of the study and the research protocol. JJvdD and DS will coordinate the study, are responsible for data acquisition and will conduct statistical analysis. MAK will provide economic statistical consultation. JJvdD, DS, PAJL, ARK, MAK, PJEB and $S M A B-Z$ were involved in the writing, editing and approval of the final manuscript.

Funding This work was supported by the Netherlands Organisation for Health Research and Development (ZonMW) (80-83600-98-3141).

Competing interests None declared.

Ethics approval The local Medical Ethics Committee of Erasmus Medical Center has approved the trial (MEC-2015-293), which is registered in the Dutch Trial registry (NTR4798) and EudractCT database (2015-001669-16).

Provenance and peer review Not commissioned; peer reviewed for ethical and funding approval prior to submission.

Open Access This is an Open Access article distributed in accordance with the Creative Commons Attribution Non Commercial (CC BY-NC 4.0) license, which permits others to distribute, remix, adapt, build upon this work non-commercially, and license their derivative works on different terms, provided the original work is properly cited and the use is non-commercial. See: http://creativecommons.org/ licenses/by-nc/4.0/
(C) Article author(s) (or their employer(s) unless otherwise stated in the text of the article) 2017. All rights reserved. No commercial use is permitted unless otherwise expressly granted.

\section{REFERENCES}

1. Sharma L, Kapoor D, Issa S. Epidemiology of osteoarthritis: an update. Curr Opin Rheumatol 2006;18:147-56.

2. Johnson VL, Hunter DJ. The epidemiology of osteoarthritis. Best Pract Res Clin Rheumatol 2014;28:5-15.

3. Netherlands statistics. CBS) Gezondheid en zorg in cijfers 2013, 2017. Accessed. http://www.cbs.nl/NR/rdonlyres/B3173C43-368C4190-8D9C-88E6BBF2CBE8/0/2013c156puberr.pdf

4. Brand C, Hunter D, Hinman R, et al. Improving care for people with osteoarthritis of the hip and knee: how has national policy for osteoarthritis been translated into service models in Australia? Int $J$ Rheum Dis 2011;14:181-90.

5. Mitchell HL, Carr AJ, Scott DL. The management of knee pain in primary care: factors associated with consulting the GP and referrals to secondary care. Rheumatology 2006;45:771-6.

6. Peat G, McCarney R, Croft P. Knee pain and osteoarthritis in older adults: a review of community burden and current use of primary health care. Ann Rheum Dis 2001;60:91-7.

7. The Dutch College of General Practitioners. NHG Standaard Niettraumatische knieklachten 2016. Accessed https://www.nhg.org/sta ndaarden/volledig/nhg-standaard-niet-traumatische-knieklachten\# Richtlijnendiagnostiek (04 July 2017).

8. da Costa BR, Nüesch E, Kasteler R, et al. Oral or transdermal opioids for osteoarthritis of the knee or hip. Cochrane Database Syst Rev 2014:CD003115.

9. Towheed TE, Maxwell L, Judd MG, et al. Acetaminophen for osteoarthritis. Cochrane Database Syst Rev 2006:CD004257.

10. Zhang W, Nuki G, Moskowitz RW, et al. OARSI recommendations for the management of hip and knee osteoarthritis: part III: Changes in evidence following systematic cumulative update of research published through January 2009. Osteoarthritis Cartilage 2010;18:476-99.

11. Conaghan PG, Porcheret M, Kingsbury SR, et al. Impact and therapy of osteoarthritis: the Arthritis Care OA Nation 2012 survey. Clin Rheumatol 2015;34:1581-8.

12. Juby AG, Skeith K, Davis P. Patients' awareness, utilization, and satisfaction with treatment modalities for the management of their osteoarthritis. Clin Rheumatol 2005;24:535-8.

13. Beswick AD, Wylde V, Gooberman-Hill R, et al. What proportion of patients report long-term pain after total hip or knee replacement for osteoarthritis? A systematic review of prospective studies in unselected patients. BMJ Open 2012;2:e000435.

14. Schaible HG. Mechanisms of chronic pain in osteoarthritis. Curr Rheumatol Rep 2012;14:549-56.

15. Mease PJ, Hanna S, Frakes EP, et al. Pain mechanisms in osteoarthritis: understanding the role of central pain and current approaches to its treatment. $J$ Rheumatol 2011;38:1546-51

16. Hochman JR, Davis AM, Elkayam J, et al. Neuropathic pain symptoms on the modified painDETECT correlate with signs of central sensitization in knee osteoarthritis. Osteoarthritis Cartilage 2013;21:1236-42.

17. Lluch E, Torres R, Nijs J, et al. Evidence for central sensitization in patients with osteoarthritis pain: a systematic literature review. Eur $J$ Pain 2014;18:1367-75.

18. Abou-Raya S, Abou-Raya A, Helmii M. Duloxetine for the management of pain in older adults with knee osteoarthritis: randomised placebo-controlled trial. Age Ageing 2012;41:646-52.

19. Chappell AS, Ossanna MJ, Liu-Seifert $\mathrm{H}$, et al. Duloxetine, a centrally acting analgesic, in the treatment of patients with osteoarthritis knee pain: a 13-week, randomized, placebo-controlled trial. Pain 2009;146:253-60.

20. The Netherlands Institute for Social Science. https://www.scp.nl/ Onderzoek/Lopend_onderzoek/A_Z_alle_lopende_onderzoeken/ Statusscores 2017

21. Smink AJ, Bierma-Zeinstra SM, Dekker J, et al. Agreement of general practitioners with the guideline-based stepped-care strategy for patients with osteoarthritis of the hip or knee: a cross-sectional study. BMC Fam Pract 2013;14:33.

22. Jm L. Physician performance: The roles of knowledge, skill, and environment. Teach Learn Med 1992;4:86-96.

23. Landon BE, Reschovsky J, Reed M, et al. Personal, organizational, and market level influences on physicians' practice patterns: results of a national survey of primary care physicians. Med Care 2001;39:889-905. 
24. Altman RD. Classification of disease: osteoarthritis. Semin Arthritis Rheum 1991;20(6 Suppl 2):40-7.

25 .The Netherlands Federation of University Medical Centres. http://www.nfu.nl/img/pdf/NFU-12.6053_Kwaliteitsborging_ mensgebonden_onderzoek_2.0.pdf 2017

26. Roos EM, Roos HP, Lohmander LS, et al. Knee Injury and Osteoarthritis Outcome Score (KOOS)--development of a selfadministered outcome measure. J Orthop Sports Phys Ther 1998;28:88-96.

27. Klässbo M, Larsson E, Mannevik E. Hip disability and osteoarthritis outcome score. An extension of the Western Ontario and McMaster Universities Osteoarthritis Index. Scand J Rheumatol 2003;32:46-51.

28. Collins NJ, Prinsen CA, Christensen R, et al. Knee Injury and Osteoarthritis Outcome Score (KOOS): systematic review and meta-analysis of measurement properties. Osteoarthritis Cartilage 2016;24:1317-29.

29. Brooks R. EuroQol: the current state of play. Health Policy 1996;37:53-72.

30. Bouwmans CA, Hakkaart-van Roijen L, Koopmanschap MA, et al. Manual of the iMTA Medical Consumption Questionnaire (iMCQ). Rotterdam: iMTA, Erasmus University Rotterdam 2013.

31. Bouwmans CA, Hakkaart-van Roijen L, Koopmanschap MA, et al. Manual of the iMTA Productivity Costs Questionnaire (iPCQ). Rotterdam: iMTA, Erasmus University Rotterdam 2013.

32. Horne R, Weinman J. Patients' beliefs about prescribed medicines and their role in adherence to treatment in chronic physical illness. $J$ Psychosom Res 1999;47:555-67.

33. Zwikker HE, van Dulmen S, den Broeder AA, et al. Perceived need to take medication is associated with medication non-adherence in patients with rheumatoid arthritis. Patient Prefer Adherence 2014;8:1635-45.

34. Menckeberg TT, Bouvy ML, Bracke M, et al. Beliefs about medicines predict refill adherence to inhaled corticosteroids. J Psychosom Res 2008;64:47-54.

35. Pham T, van der Heijde D, Altman RD, et al. OMERACT-OARSI initiative: Osteoarthritis Research Society International set of responder criteria for osteoarthritis clinical trials revisited. Osteoarthritis Cartilage 2004;12:389-99.

36. Hochman JR, Gagliese L, Davis AM, et al. Neuropathic pain symptoms in a community knee OA cohort. Osteoarthritis Cartilage 2011;19:647-54
37. Moss P, Benson HA, Will R, et al. Patients With Knee Osteoarthritis Who Score Highly on the PainDETECT Questionnaire Present With Multi-modality Hyperalgesia, Increased Pain and Impaired Physical Function. Clin J Pain 2017:1.

38. Rienstra W, Blikman T, Mensink FB, et al. The Modified painDETECT Questionnaire for Patients with Hip or Knee Osteoarthritis: Translation into Dutch, Cross-Cultural Adaptation and Reliability Assessment. PLoS One 2015;10:e0146117.

39. Zigmond AS, Snaith RP. The hospital anxiety and depression scale. Acta Psychiatr Scand 1983;67:361-70.

40. Rozendaal RM, Koes BW, van Osch GJ, et al. Effect of glucosamine sulfate on hip osteoarthritis: a randomized trial. Ann Intern Med 2008;148:268-77.

41. Adams G, Gulliford MC, Ukoumunne OC, et al. Patterns of intracluster correlation from primary care research to inform study design and analysis. J Clin Epidemiol 2004;57:785-94.

42. Staniszewska S, Haywood KL, Brett J, et al. Patient and public involvement in patient-reported outcome measures: evolution not revolution. Patient 2012;5:79-87.

43. Lamers LM , et al. Measuring the quality of life in economic evaluations: the Dutch EQ-5D tariff] Kwaliteit van leven meten in economische evaluaties: het Nederlands EQ-5D-tarief. Ned Tijdschr Geneeskd 2005;149:1574-8.

44. Chappell AS, Desaiah D, Liu-Seifert $H$, et al. A double-blind, randomized, placebo-controlled study of the efficacy and safety of duloxetine for the treatment of chronic pain due to osteoarthritis of the knee. Pain Pract 2011;11:33-41.

45. Frakes EP, Risser RC, Ball TD, et al. Duloxetine added to oral nonsteroidal anti-inflammatory drugs for treatment of knee pain due to osteoarthritis: results of a randomized, double-blind, placebocontrolled trial. Curr Med Res Opin 2011;27:2361-72.

46. Tunis SR, Stryer DB, Clancy CM. Practical clinical trials: increasing the value of clinical research for decision making in clinical and health policy. JAMA 2003;290:1624-32.

47. Allen KD, Bierma-Zeinstra SM, Foster NE, et al. OARSI Clinical Trials Recommendations: Design and conduct of implementation trials of interventions for osteoarthritis. Osteoarthritis Cartilage 2015;23:826-38. 